werden ausführliche Fallstudien zu einzelnen Ländern vorgestellt. Es zeigt sich, dass demokratische Systeme mit einer funktionierenden Verwaltung stets am besten dastehen. Norris schlussfolgert, was sie auch schon zum Einstieg ihrer Arbeit gewissermaßen als Prämisse formuliert hat: Es ist nicht ausreichend, nur einen Schwerpunkt in Reformen zu verfolgen. „A fine balance needs to be struck so that democracy and state capacity are developed simultaneously“ (S. 192).

In der Zusammenschau unterschiedlicher aktueller Texte zeigt sich dreierlei: Der Governance-Begriff bleibt mehrdeutig und wird sehr vielfältig verwendet. Folglich muss zum Verständnis politisch-administrativer Prozesse der Blick über die klassischen organschaftlichen Akteure hinausgehen - was aber keine bahnbrechende Erkenntnis ist. Und schließlich betont er die Bedeutung des Informalen neben dem Formalen; auch dies ist für Politikwissenschaftler nicht neu. Eine Gefahr besteht in der normativen Überhöhung des Begriffes und in der analytischen Umkehrung der Diagnose: Auch wenn Staatlichkeit sich wandelt, ist sie zu einem Großteil weiterhin in klassischer Form wirksam. Ohne Frage hilft der Governance-Begriff als analytische Brille, die Realität wahrzunehmen - doch darf man nicht vergessen, auch die „alten“ Brillen wie Gewaltenteilung, Demokratie, Macht aufzusetzen. Aus dieser Kombination erhält man am ehesten ein geschärftes Bild der sich ständig verändernden Realität.

Sven T. Siefken

\title{
Regieren in Partnerschaft - eindrucksvoller Blick hinter die Kulissen in Paris und Bonn
}

Waechter, Matthias: Helmut Schmidt und Valéry Giscard d'Estaing - Auf der Suche nach Stabilität in der Krise der 70er Jahre, Studien der Helmut und Loki Schmidt-Stiftung, Band 6, Edition Temmen, Bremen 2011, 168 Seiten, € 14,90.

Gut ein Jahr vor dem 50. Jahrestag der Unterzeichnung des deutsch-französischen Kooperationsvertrages am 22. Januar 1963 („Élysée-Vertrag“) legte Matthias Waechter, Direktor des Nizzaer Institut des Hautes Études Internationales und Privatdozent am Historischen Seminar der Universität Freiburg, eine 168-seitige Studie über das Tandem Helmut Schmidt und Valéry Giscard d'Estaing vor. Beide gehören neben Charles de Gaulle und Konrad Adenauer sowie François Mitterrand und Helmut Kohl zu den Architekten der binationalen Zusammenarbeit. Beide Staatsmänner übernahmen nahezu gleichzeitig die wichtigsten politischen Ämter in ihren Ländern: Schmidt nach Willy Brandts Rücktritt im Mai 1974 und Giscard d'Estaing nach Georges Pompidous Tod im Juni desselben Jahres. Schon vorher hatten sie sich als Finanzminister kennen- und schätzen gelernt. Das Ende ihrer Amtszeiten fiel auch nicht weit auseinander: Mai 1981 für den Staatspräsidenten und Oktober 1982 für den Bundeskanzler.

Gegliedert ist Waechters verdienstvolles Werk in fünf Kapitel. Nach der Einleitung beleuchtet er den persönlichen und politischen Werdegang beider: Der Deutsche stammte aus kleinbürgerlichem Milieu, sein französischer Partner aus dem Großbürgertum mit Schloss in der Auvergne. Der erste Hauptteil der Studie firmiert unter dem Titel „Kooperation in der internationalen Krise“ nach dem ersten Ölpreisschock im Herbst 1973. Beide Staatsmänner wurden „von unterschiedlichen öffentlichen Erwartungen und Wahrnehmungen 
begleitet. Der eine verkörpert weitreichende Hoffnungen auf eine gesellschaftliche Modernisierung, während [der Kanzler], als Krisenmanager geschätzt, die anbrechende Wirtschaftsrezession aufzuhalten verspricht" (S. 51). Waechter zeichnet die Linie von Giscard d'Estaings „changement"-Bemühungen nach, das Land aus den Verkrustungen der gaullistischen Epoche zu lösen, die Wirtschaft zu modernisieren, die beginnende Arbeitslosigkeit einzudämmen und einschneidende Reformen im gesellschaftspolitischen Bereich (zum Beispiel Schwangerschaftsabbruch, rechtliche Gleichstellung der Ehepartner) durchzusetzen. Ein Hauptproblem, unter dem Frankreich bis zum heutigen Tag leidet, ist die hohe strukturelle Jugendarbeitslosigkeit, die sich Ende der 1970er Jahre zunehmend bemerkbar machte. Auch Schmidt hatte mit ähnlichen Problemen zu kämpfen. Hinzu kam ein Anstieg der Inflationsraten in beiden Ländern. Die wirtschaftlichen und finanziellen Probleme begleiteten beide Politiker während ihrer gesamten Amtszeit.

Beide nutzten die Konsultationsmechanismen des Freundschaftsvertrages, um rasch ein Höchstmaß an Kooperation und gegenseitiger Abstimmung zu vereinbaren. Waechter zitiert Schmidt, wenn dieser darauf verweist, dass zwischen den höchsten Entscheidungsträgern der Staaten „ein möglichst enger, direkter Meinungsaustausch und - nach Möglichkeit - ein hoher Grad an gegenseitigem Vertrauen erzielt [wird], um zu konkreten Ergebnissen zu gelangen" (S. 58). Dies ist beiden zweifellos gelungen: die engen Abstimmungen vor jedem Treffen der damals neun Mitglieder der Europäischen Gemeinschaft, die Schaffung des Vorläufers der heutigen gemeinsamen Währung, des Europäischen Währungssystems EWS, und die regelmäßigen Zusammenkünfte der führenden westlichen Wirtschaftsmächte im Rahmen der G7-Treffen.

Welche Gründe haben Giscard d'Estaing zu einer so engen Zusammenarbeit mit Schmidt bewogen? Warum räumte er dem deutsch-französischen Bündnis höchste Priorität ein? Laut Waechter bekundete der Staatschef, dass nur „,im Einklang mit der Bundesrepublik Deutschland die von ihm so stark gewünschten Fortschritte in der europäischen Einigung erzielt werden“, beide Länder verbinde eine "Schicksalsgemeinschaft" (S. 60). Der "gemeinsame Motor" stieß bei den europäischen Partnern auf ein so großes Vertrauen, dass beide die übrigen jeweils bei ihren Plänen mitzuziehen vermochten. Für Giscard d'Estaing stand fest, dass seinem Land im geeinten Europa die Führungsrolle zufiel. Schmidt akzeptierte dies und schrieb später in seinen Memoiren: Selbst wenn er, Schmidt, die Idee hatte, überließ er aus historischen Gründen immer seinem Partner den Vortritt. Ein tiefes Vertrauensverhältnis verband beide, das - neben den häufigen privaten Treffen - auch auf den nahezu wöchentlichen Telefonaten über die Europa- und Weltpolitik fußte.

Trotz der engen Zusammenarbeit blieben Interessenunterschiede nicht aus. Im zweiten Hauptkapitel „Grenzen der Konvergenz" werden diese thematisiert. Beispielhaft sei auf den Gegensatz im strategischen Bereich verwiesen. Waechter arbeitet die unterschiedlichen Positionen klar heraus. Während die Nato und das Bündnis mit den USA für die Bundesrepublik lebenswichtig waren beziehungsweise sind, setzte Giscard d'Estaing die von de Gaulle begründete "Ideologie der Unabhängigkeit" (so Schmidt) fort. Die Atomstreitmacht des Nachbarlandes stelle, da sie außerhalb der westlichen Allianz bleibe, für die Bundesrepublik eher ein Risiko als einen Schutzfaktor dar, so der Kanzler (S. 125). Folglich blieb die beiderseitige Sicherheitspolitik aus der Zusammenarbeit weitgehend ausgeklammert (erst Mitterrand aktivierte diesen schon 1963 vertraglich vereinbarten Bereich). Dass die westlich des Vogesenkamms stationierten atomaren Kurzstreckenraketen höchste Besorgnis in Bonn verursachten, war kein Geheimnis. 
Auch die beinahe chronische Germanophobie vieler französischer Intellektueller vor allem nach der Verurteilung der Baader-Meinhof-Bande wird von Waechter thematisiert (S. 148). Der damals immer wiederkehrende Verweis auf die deutsche Nazi-Vergangenheit erfülle, so zitiert der Autor den exzellenten französischen Deutschlandkenner Joseph Rovan, in Frankreich die Funktion, historische Schuld auf das Nachbarland abzuladen und unter anderem von eigenen Verbrechen während des Algerienkrieges abzulenken (S. 149). Bonn erwog damals übrigens eine Öffentlichkeitsoffensive, um nach Jean-Paul Sartres Besuch bei Andreas Baader im Hochsicherheitstrakt des Gefängnisses Stuttgart-Stammheim das negative Deutschlandbild in Frankreich korrigieren zu helfen.

Waechter gelingt es mit seinem auch reich bebilderten Buch, dem Leser einen eindrucksvollen Blick hinter die Kulissen der deutsch-französischen Beziehungen in dieser Epoche zu ermöglichen. Studien in Schmidts Privatarchiv sowie Gespräche mit beiden Staatsmännern erlaubten es ihm, nahezu ein Jahrzehnt grenzüberschreitender enger, freundschaftlicher Partnerschaft authentisch und anschaulich aufzuarbeiten.

Udo Kempf

\title{
Regieren als strategische Steuerung - empirisch gesättigter Vergleich von Berlin und London
}

\author{
Tils, Ralf: Strategische Regierungssteuerung. Schröder und Blair im Vergleich, VS-Verlag für \\ Sozialwissenschaften, Wiesbaden 2011, 417 Seiten, € 29,95.
}

Mit Strategiefragen hat sich die Politikwissenschaft lange Zeit nur am Rande beschäftigt. Die Themenfelder politische Steuerung und politische Führung fristeten ein Schattendasein - wohl auch, weil die Disziplin ihre Aufmerksamkeit eher der Erforschung der Mechanismen der Machtkontrolle und -balance widmete und das „Regieren“ selber vernachlässigte. Dies hat sich in den letzten beiden Dekaden deutlich verändert. Zu den zahlreichen Studien, die im Bereich der Regierungsforschung erschienen sind, gehört auch die Habilitationsschrift von RalfTils, in der er die Steuerungsleistungen zweier sozialdemokratisch geführter Regierungen vergleicht. Tils ist im Themengebiet „Strategische Steuerung“ eine bekannte Größe in der Politikwissenschaft. So hat er - teilweise zusammen mit seinem Vater Joachim Rasch$k e$ - mehrere Monographien beziehungsweise Sammelbände zum Thema veröffentlicht. Die Habilitationsschrift rundet diese insofern ab, als dass nun erstmals eine komparative Betrachtung vorgelegt wird. Im Rahmen dieser Untersuchung analysiert der Autor die Regierung von Gerhard Schröder (1998 bis 2005) und von Tony Blair (1997 bis 2005).

In den ersten drei Abschnitten führt er in das Konzept der strategischen Steuerung ein und entwickelt für einen analytischen Bezugsrahmen den Vergleich. Hierauf folgen Kapitel zu den Strategieprofilen der beiden Regierungen, in denen jeweils auf eine dichte Beschreibung der Eigenheiten in einem zweiten Schritt fallspezifische Erklärungen geliefert werden. Abschließend werden die beiden Fälle systematisch gegenübergestellt und Erklärungen für die Unterschiede geliefert. Der politikwissenschaftliche Mehrwert dieser Studie liegt im theoretischen wie im empirischen Bereich. Eine genuine Eigenleistung liegt zunächst in der Entwicklung von Begriffen und Kategorien, mit denen sich die politische Strategie beschreiben und rekonstruieren lässt. So analysiert Tils die Voraussetzungen und Bedingungen der 